\title{
Leiomyosarcoma of the inferior vena cava: Clinical experience with four cases

\author{
Said Abisi ${ }^{1}$, Gareth J Morris-Stiff ${ }^{1}$, David Scott-Coombes ${ }^{1}$, Ian M Williams ${ }^{1}$, \\ Anthony G Douglas-Jones ${ }^{2}$ and Malcom C Puntis*1
}

\begin{abstract}
Address: ${ }^{1}$ Department of Surgery, University Hospital of Wales, Heath Park, Cardiff, CF14 4XN, UK and ${ }^{2}$ Department of Pathology, University Hospital of Wales, Heath Park, Cardiff, CF14 4XN, UK

Email: Said Abisi - said.abisi@kcl.ac.uk; Gareth J Morris-Stiff - garethmorrisstiff@hotmail.com; David Scott-Coombes - David.ScottCoombes@cadiffandvale.wales.nhs.uk; Ian M Williams - Ian.Williams@cadiffandvale.wales.nhs.uk; Anthony G Douglas-Jones - douglasjones@cf.ac.uk; Malcom C Puntis* - Malcolm.Puntis@cadiffandvale.wales.nhs.uk

* Corresponding author
\end{abstract}

Published: 04 January 2006

World Journal of Surgical Oncology 2006, 4:I doi:10.1 I86/1477-78/9-4-1

This article is available from: http://www.wjso.com/content/4/I/I

(c) 2006 Abisi et al; licensee BioMed Central Ltd.

This is an Open Access article distributed under the terms of the Creative Commons Attribution License (http://creativecommons.org/licenses/by/2.0), which permits unrestricted use, distribution, and reproduction in any medium, provided the original work is properly cited.

\begin{abstract}
Background: Leiomyosarcoma of the inferior vena cava is a rare tumor that presents in an insidious manner with non-specific symptoms. Given its rarity, there are no consensus guidelines to its management. The aim of this study was to report the clinical experience in the management of patients presenting to our institution during a 12 year period.
\end{abstract}

Patients and Methods: Four patients with leiomyosarcomas of the inferior vena cava were managed at our institution during the period reviewed. Patient details were identified through a search of the pathology department computerized database, and case notes were retrospectively reviewed to obtain details of presentation and management.

Results: There were 3 females and I male with a mean age of 59 years. All tumors were identified within 2 months of first symptoms. Three of the 4 had localized tumors whilst I patient had lung metastases at presentation. The three patients with resectable tumors underwent radical surgical excision of the tumor, and two patients had postoperative radiotherapy. One patient died of recurrence at 7 months, and another at 30 months. The third patient is currently well and disease free at 16 months. The fourth patient with metastatic disease was treated with chemotherapy alone and survived 36 months.

Conclusion: Leiomyosarcoma of the inferior vena cava is an uncommon tumor that presents with non-specific symptoms. At the time of presentation, tumors are usually large and resection is challenging but probably offers the best opportunity for long-term survival.

\section{Background}

Leiomyosarcomas of the inferior vena cava (IVC) are rare as illustrated by the fact that an International IVC Leiomyosarcoma registry established in 1992 collected, by means of literature review and personal communication, only
218 cases by the time of publication of its first report in 1996 [1]. Given their anatomical location, it is not surprising that they often present insidiously with non-specific symptoms such as: abdominal pain, weight loss, mass, fever, weakness, anorexia, vomiting, night sweats, dysp- 
Table I: Demographic features and presenting symptoms of patients with IVC leiomyosarcoma.

\begin{tabular}{|c|c|c|c|c|}
\hline & \multicolumn{4}{|c|}{ Patients } \\
\hline & $\mathbf{I}$ & 2 & 3 & 4 \\
\hline \multicolumn{5}{|l|}{ Demographics } \\
\hline Sex & Female & Female & Male & Female \\
\hline Age & 57 & 60 & 57 & 61 \\
\hline \multicolumn{5}{|l|}{ Symptoms \& Signs } \\
\hline Abdominal Pain & Yes & Yes & No & Yes \\
\hline Weakness & No & Yes & Yes & Yes \\
\hline Anorexia & No & Yes & No & Yes \\
\hline Vomiting & No & No & No & Yes \\
\hline Night sweats & No & No & No & No \\
\hline Dyspnoea & No & No & No & No \\
\hline Mass & No & No & No & No \\
\hline Fever & No & No & No & No \\
\hline $\begin{array}{r}\text { Abdominal wall veins } \\
\text { distension }\end{array}$ & Yes & No & No & No \\
\hline \multicolumn{5}{|l|}{ Diagnosis } \\
\hline Modalities used & USS + CT & USS + CT & $\mathrm{CT}$ & USS + CT \\
\hline Location & Lower & Middle & Upper & Middle \\
\hline Size & $8 \times 14 \mathrm{~cm}$ & $8 \times 7 \mathrm{~cm}$ & $10 \times 10.5 \mathrm{~cm}$ & $9.5 \times 7 \mathrm{~cm}$ \\
\hline Embolus present & No & No & Yes & No \\
\hline Metastasis present & Yes (Lung) & No & No & No \\
\hline Biopsy performed & Yes & No & No & No \\
\hline
\end{tabular}

USS - ultrasound scan; CT - computed tomography

noea, or they may present with Budd-Chiari syndrome $[2,3]$.

IVC Leiomyosarcomas are classified anatomically according to their relationship to the liver and renal vessels: lower or Segment I (below the renal vessels); middle or Segment II (renal vessels to retrohepatic IVC) and upper or Segment III (suprahepatic IVC) [4]. The management of these tumors has been, where possible, by means of radical en-bloc resection with a view of achieving clear margins. However, in spite of an overall 50\% 5-year survival, half of the tumors recur either locally or metastasize $[1,3]$.

We present our experience with four cases of IVC leiomyosarcoma managed at our institution between January 1993 and December 2004.

Table 2: Management and outcome data for patients with IVC leiomyosarcoma.

\begin{tabular}{|c|c|c|c|c|}
\hline & \multicolumn{4}{|c|}{ Patients } \\
\hline & $\mathbf{I}$ & 2 & 3 & 4 \\
\hline \multicolumn{5}{|l|}{ Management } \\
\hline Operation & No & $\begin{array}{c}\text { Resection and IVC PTFE } \\
\text { Patch }\end{array}$ & $\begin{array}{l}\text { Resection and primary } \\
\text { repair }\end{array}$ & $\begin{array}{c}\text { Resection and IVC Vein } \\
\text { Patch }\end{array}$ \\
\hline Chemotherapy & Yes & No & No & No \\
\hline Adjuvant therapy & None & Radiotherapy & None & Radiotherapy \\
\hline Histological grade & High & High & High & High \\
\hline \multicolumn{5}{|l|}{ Complications } \\
\hline Limb oedema & NA & No & No & No \\
\hline Venous insufficiency & NA & No & No & No \\
\hline Other & None & No & CVA & No \\
\hline \multicolumn{5}{|l|}{ Follow-up } \\
\hline Recurrence & - & No & Yes (5 months) & No \\
\hline Status & Dead & Dead & Dead & Alive \\
\hline Survival & 36 months & 30 months & 7 months & 16 months \\
\hline
\end{tabular}

NA - not applicable; CVA - cerebrovascular accident 


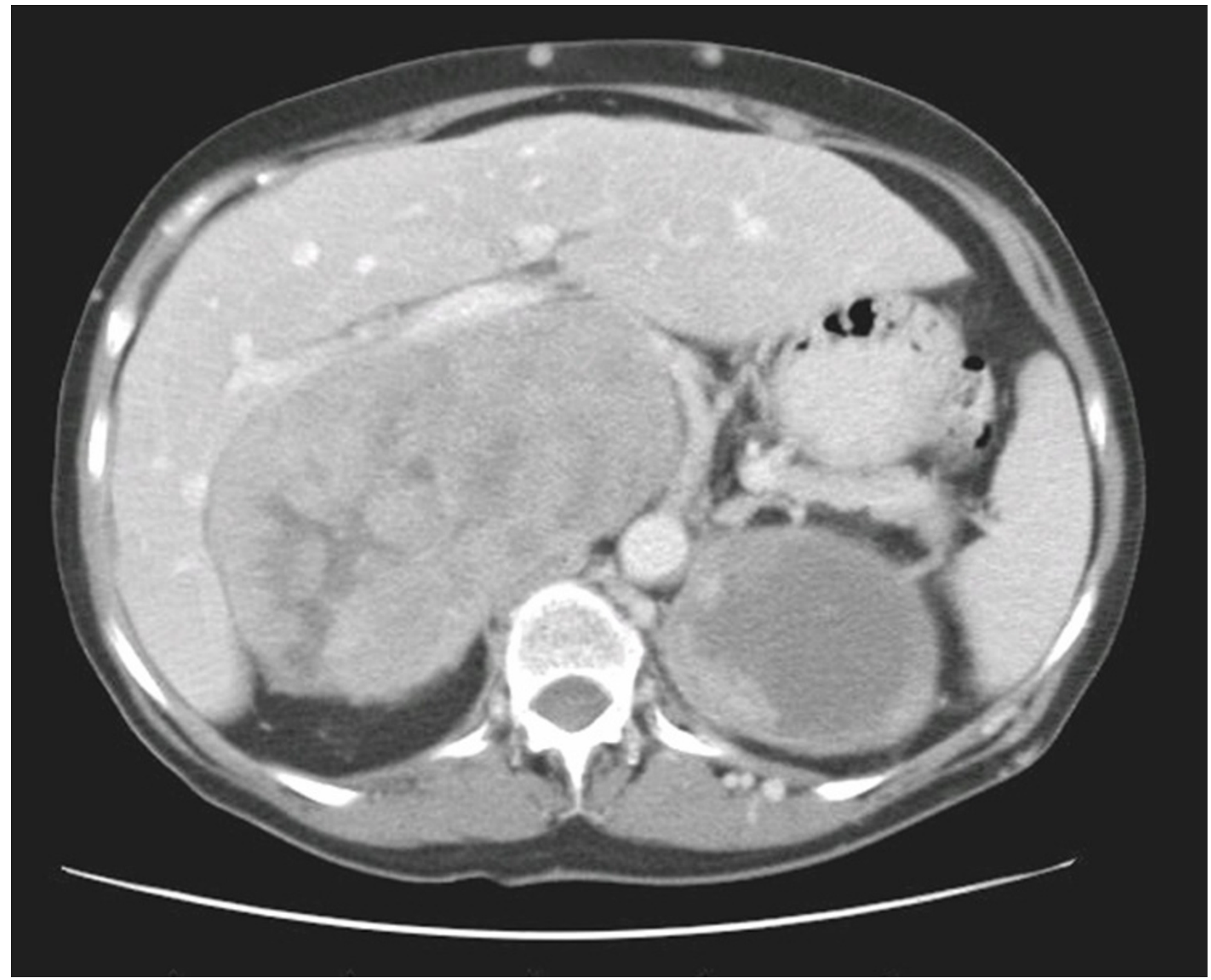

Figure I

Computerized tomographic scan of patient I demonstrating a large tumor.

\section{Patients and methods}

From January 1993 to December 2004, 4 patients were diagnosed with leiomyosarcomas of the IVC. There were 3 females and 1 male with a mean age of 59 years (range: 57-61 years). The demographic details and symptoms at presentation are summarized in Table 1.

Clinical data was obtained from review of the patient notes, whilst radiological and histological results were obtained from prospectively maintained departmental computerized databases.

\section{Results}

All 4 patients were symptomatic at presentation although there was significant variation in symptoms between patients (Table 1). Patient 1 was referred specifically for investigation of distended abdominal wall veins.

The diagnosis of a caval tumor was made on an ultrasound scan (USS) in 3 of the 4 cases and on computed tomography (CT) in the fourth. CT scans were performed to further characterize the origin of the tumors, demonstrate any contiguous invasion, and to exclude extraabdominal metastases (Figure 1). Patient 3 was diagnosed with a tumor embolus to the right atrium preoperatively. No further preoperative imaging was performed. Given the location of the tumors, and based on experience with other sarcomas, no biopsy was performed preoperatively for the 3 patients undergoing surgical excision. Patient 1 underwent biopsy of the primary tumor confirm the diagnosis prior to commencement of chemotherapy. 


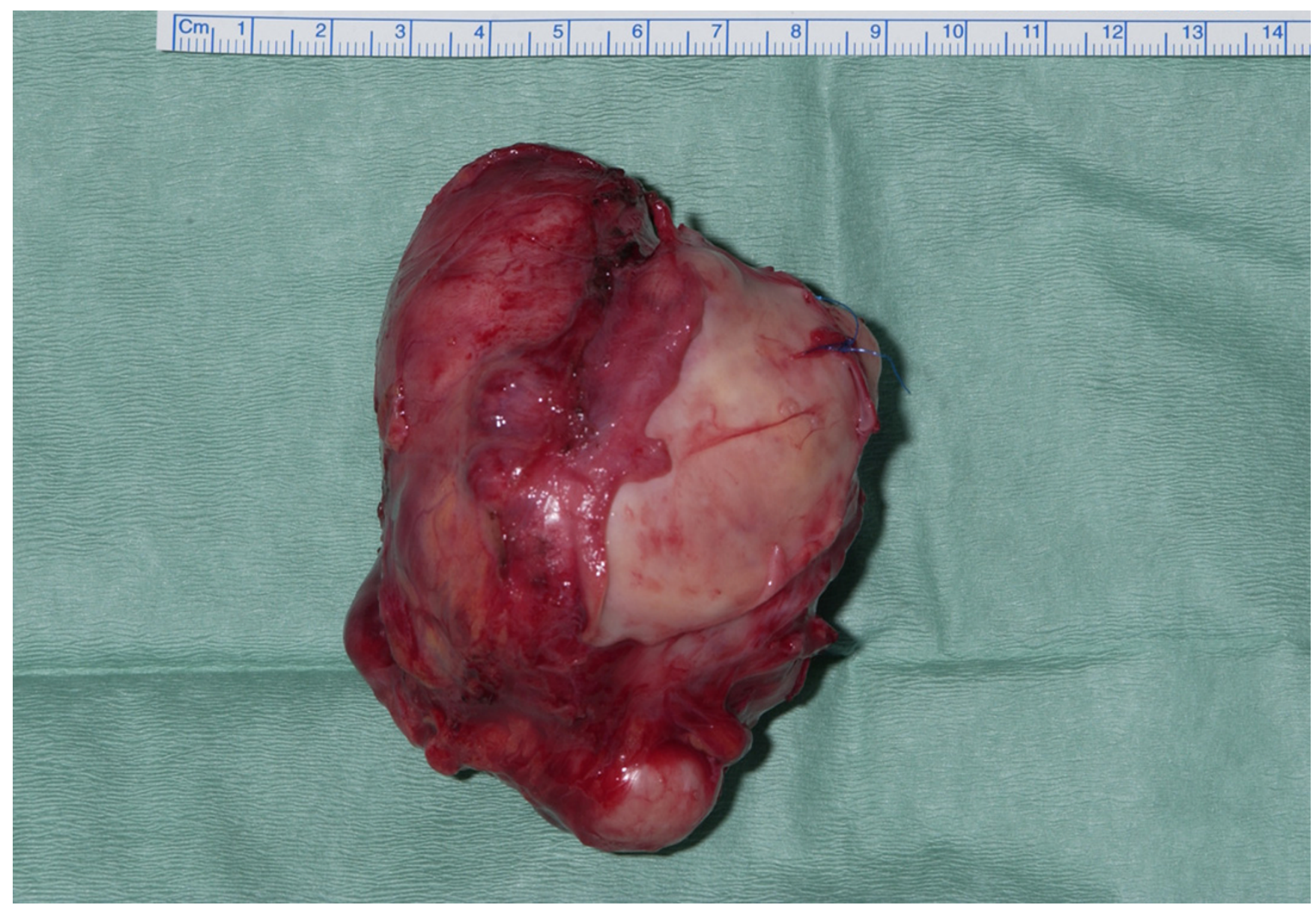

\section{Figure 2}

Gross appearance of the tumor.

Of the patients who underwent laparotomy, the tumor was excised en-bloc in all 3 cases. Patient 3 also underwent a thoracotomy and excision of a tumor embolus from the right atrium. In this patient, the point of origin of the tumor on the IVC was narrow and there was enough mobility to primarily close the IVC. Whilst in the remaining 2 cases, the tumor had a broad base. In these cases, the tumor was excised with a generous cuff of normal IVC wall, the defect was patched with a portion of long saphenous vein in patient 4 (Duplex ultrasound was used preoperatively to demonstrate the absence of deep venous thromboses, and deep venous incompetence in the lower limbs. In-patient 2 a PTFE patch was used to repair the IVC defect.

All tumors were large volume masses and had the classical tan-colored, nodular appearance (Figure 2). Histological examination revealed all to be high-grade leiomyosarcomas composed of spindle cells with positive immunohistochemistry staining for Desmin, HHF 35(muscle specific actin), SMA (smooth muscles actin), and negative for epi- thelial marker AE1/AE3, S100 protein confirming smooth muscles origin, and excluding nerve sheath origin (Figure $3)$.

The postoperative course was unremarkable for 2 patients. Patient 3, however, suffered a cerebrovascular accident perioperatively, but subsequently made a full recovery. Importantly, there were no complications in relation to the venous blood flow in the inferior vena cava and no renal impairment. Two of the patients (patient 2 and 4) undergoing resection received postoperative radiotherapy.

Patient 1 had a variety of chemotherapy protocols as well as palliative therapy, and survived 36 months. Disease recurrence occurred in patients 2 and 3. Patient 3 presented with widespread metastases and died at 7 months, whilst patient 2 developed disseminated disease 2 years after his surgery and died at 30 months post resection. Patient 4 is alive and disease free at 16 months post surgery. 


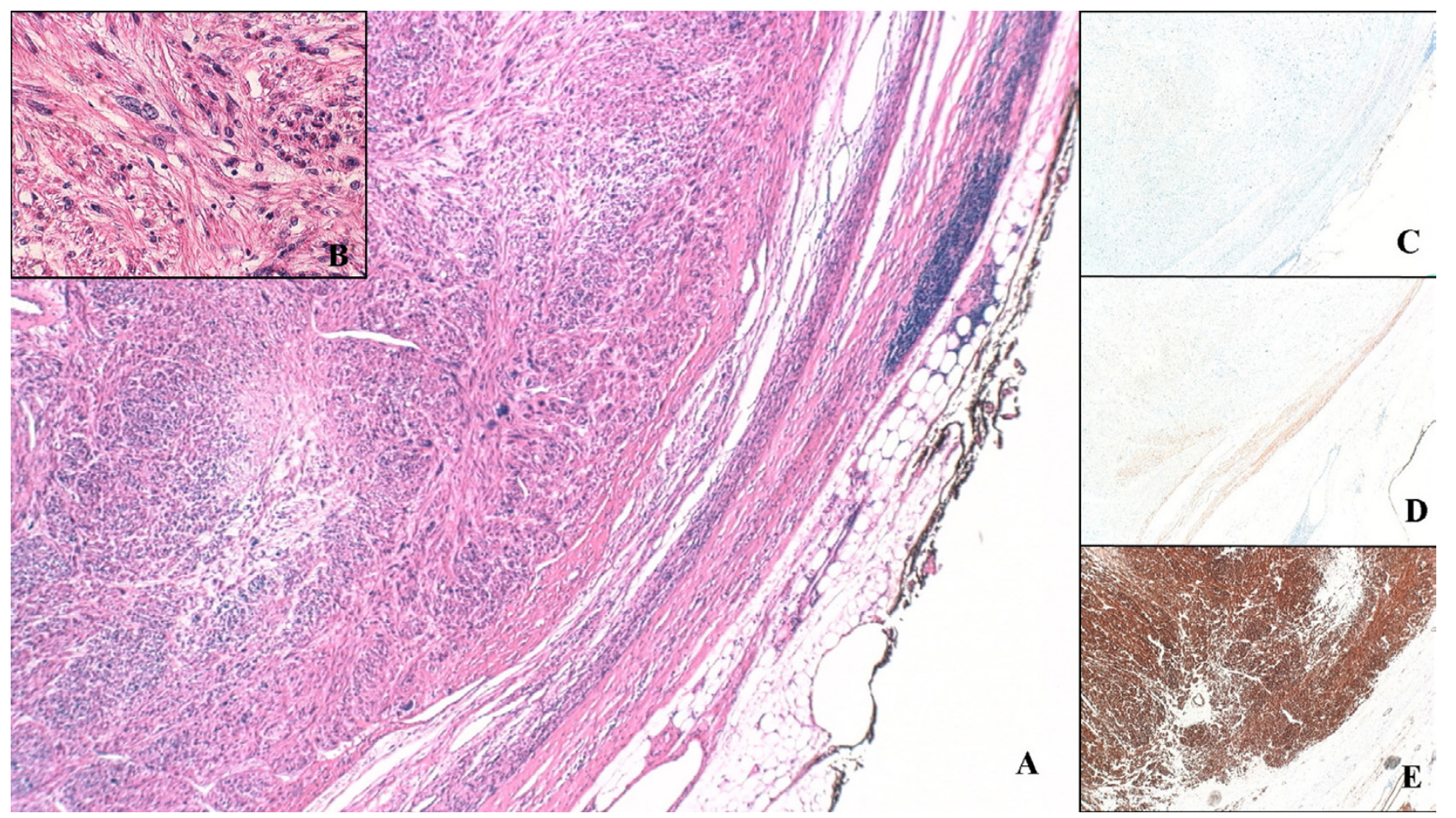

\section{Figure 3}

Photomicrograph of the edge of the tumour $(\times 40)$. (A). The tumour is composed of spindle cells which show nuclear pleomorphism with occasional giant nuclei (Hematoxylin and eosin $\times 200$ ), (B). Immunohistochemistry for epithelial marker AEI/AE3 (C), and for SIO0 (D) is negative, but tumor is strongly positive for smooth muscle marker smooth muscle actin(E).

\section{Discussion}

The first reported case of leiomyosarcoma of the inferior vena cava was documented in the German literature by Perl [5] in 1871 but as of 1996 only 218 had been collected [1]. Although only $2 \%$ of leiomyosarcomas are vascular in origin, tumors of the IVC account for at least half the cases [4]. The majority of cases (75-90\%) occur in women [4].

Leiomyosarcomas of the IVC are rare malignant tumors originating from the smooth muscle cells of the media of the cava and demonstrate three growth patterns - extraluminal, intraluminal or both [6]. The tumors are encapsulated, consisting of lobulated whorls, and histological examination demonstrates typical spindle-shaped bundles of cells with high mitotic activity and positive staining for desmin, HHF35, vimentin and smooth muscle actin.

This tumor commonly presents with a few months history of vague upper abdominal symptoms including pain, anorexia and vomiting; but may also present with more distinct signs such as an abdominal mass, deep venous thrombosis due to IVC occlusion, or less commonly as an acute Budd-Chiari Syndrome. However metastasis may be the first presentation of the tumor such as in case 1 .

Given the rarity of the tumor, and in the absence of symptoms and signs of IVC compression, the identification of an IVC leiomyosarcoma is often an unexpected finding on imaging with USS or CT. In 3 of the 4 cases in this series, the tumor was identified on USS and a CT scan was performed to determine the origin, evaluate any local invasion of the tumor and exclude pulmonary metastases. In all 3 cases undergoing surgery, a diagnosis of leiomyosarcoma was suspected preoperatively. Other imaging modalities that aid the diagnosis of IVC sarcomas include vena cavography, which allows biopsy of the tumor. Nevertheless, in some cases, the precise origin of the tumor cannot be confirmed preoperatively. Furthermore, percutaneous biopsy to confirm histology of tumors in this region is often not possible due to the anatomical location of the IVC. Thus the surgeon is often faced with a tumor of uncertain type and origin at laparotomy.

Whilst there is no strong evidence base for the management of IVC leiomyosarcomas, the treatment of choice based on the available literature is radical en-bloc surgical 
excision with a view to obtaining negative resection margins. A number of techniques have been reported for dealing with the IVC following excision including IVC ligation; primary repair of IVC; patching of IVC and interposition grafting with a synthetic conduit. In the largest single institute series of 25 patients over a 20 year period, Hollenbeck et al [7], concluded that primary repair was the method of choice where applicable as this was associated with the lowest complication rate. In our limited experience, patching of the IVC with a segment of long saphenous vein allowed adequate tumor resection without constricting the IVC. In the single case in which a primary repair was performed, tumor recurred although it is uncertain as to whether a wider resection and caval reconstitution would have changed the outcome.

When radical surgery is performed, 5- and 10-year results of $49.4 \%$ and $29.5 \%$ respectively have been reported [1]. However, recurrence rates are as high as $50 \%$ and thus surgery may simply be providing palliation in many cases [3]. Tumors of the retrohepatic IVC tend to present earlier as a result of pressure exerted on surrounding structures and as such have a superior prognosis with 5- and 10-year survival rates of $56.7 \%$ and $47.3 \%$ [1]. Other favorable factors identified by the registry include abdominal pain, the presence of a mass and the ability to achieve clear resection margins whereas poor prognostic indicators include high-grade tumors, suprahepatic tumors, and presentation with IVC occlusion or Budd-Chiari syndrome.

The role of chemotherapy and radiotherapy in the treatment of IVC leiomyosarcoma, either as a primary modality or as an adjuvant, remains to be proven. In this series, different chemotherapy protocols provided good palliation for a patient presenting with advanced disease. In the remaining three patients, only two underwent radiotherapy with a view to obtaining local control and prevention of local recurrence.

\section{Conclusion}

Whilst IVC leiomyosarcomas are certainly rare, long-term survival depends on obtaining an early diagnosis and performing extensive surgery. The more frequent use of CT in the investigation of patients with abdominal symptoms may aid the earlier diagnosis of these tumors.

\section{Competing interests}

The author(s) declare that they have no competing interests.

\section{Authors' contributions}

SA: writing the article and literature review

GJM: writing the discussion and searching the literature
DS: operative procedure and care of one of the studied cases

IW: reviewing the article and operative procedure and care of one of the studied cases

AGD: contributing the pathological aspects of the manuscript

MCP: overall supervision of the article writing

\section{Acknowledgements}

The fourth patients consent was obtained for publication of this case report.

\section{References}

I. Mingoli A, Cavallaro A, Sapienza P, Di Marzo L, Feldhaus RJ, Cava N: International registry of inferior vena cava leiomyosarcoma: analysis of a world series on 218 patients. Anticancer Res 1996, 16:320I-3205.

2. Shindo S, Matsumoto H, Ogata K, Katahira S, Kojima A, lyori K, Ishimoto T, Kobayashi M, Tada Y, Suzuki T, Itakura J, lizuka H, Matsumoto $Y$ : Surgical treatment of retroperitoneal leiomyosarcoma invading the inferior vena cava: report of three cases. Surg Today 2002, 32:929-933.

3. Mingoli A, Feldhaus RJ, Cavallaro A, Stipa S: Leiomyosarcoma of the inferior vena cava: analysis and search of world literature on 141 patients and report of three new cases. J Vasc Surg 1991, 14:688-699.

4. Bower TC, Stanson A: Diagnosis and management of tumours of the inferior vena cava. In Vascular Surgery Edited by: Rutherford RB. Philadelphia: WB Saunders Company; 2000:2077-2092.

5. Perl L: Ein fall von sarkom der vena cava inferior. Virchows Arch I87I, 53:378-383.

6. Kulaylat MN, Karakousis CP, Doerr RJ, Karamanoukian HL, O'Brien J, Peer R: Leiomyosarcoma of the inferior vena cava: a clinicopathologic review and report of three cases. J Surg Oncol 1997 , 65:205-217.

7. Hollenbeck ST, Grobmyer SR, Kent KC, Brennan MF: Surgical treatment and outcomes of patients with primary inferior vena cava leiomyosarcoma. J Am Coll Surg 2003, 197:575-579.
Publish with BioMed Central and every scientist can read your work free of charge

"BioMed Central will be the most significant development for disseminating the results of biomedical research in our lifetime. " Sir Paul Nurse, Cancer Research UK

Your research papers will be:

- available free of charge to the entire biomedical community

- peer reviewed and published immediately upon acceptance

- cited in PubMed and archived on PubMed Central

- yours - you keep the copyright

Submit your manuscript here:

http://www.biomedcentral.com/info/publishing_adv.asp
BioMedcentral 